\title{
TEM Investigation on Eutectic Phase Formation in Ni-30Cr Filler Metal 52XL
}

\author{
Cheng-Han Li, Meiyue Shao, Carolin Fink, John C. Lippold, Joerg R. Jinschek
}

Department of Materials Science and Engineering, The Ohio State University, Columbus, Ohio, USA

Eutectic phase formation in the weld metal microstructure of Tantalum (Ta)-bearing Ni-based filler metal was investigated in this work. The eutectic constituents in the interdendritic regions composed of the gamma $(\gamma)$ phase (Ni-matrix), Ta-rich carbide phase (MC) and the Cr- and Mo-rich Laves phase were identified using scanning / transmission electron microscopy (S/TEM). In addition, the orientation relationship between precipitates and matrix and element segregation were studied by electron diffraction (ED) and X-ray energy dispersive spectrum (EDS) mapping. The atomic structure of each phase and the structure of the interfaces were visualized in high-resolution STEM imaging and the stoichiometric formula of the observed Laves phase and MC carbide were determined.

$\mathrm{Ni}-30 \mathrm{Cr}$ alloys are widely used in the nuclear power industry to mitigate primary water stress corrosion cracking (PWSCC) in repair and new construction applications. Current filler metals are alloyed with niobium $(\mathrm{Nb})$ to provide resistance to ductility-dip cracking (DDC). Niobium additions promote the $\gamma / \mathrm{NbC}$ eutectic reaction at the end of solidification, which results in tortuous weld metal grain boundaries and inhibits DDC formation by grain boundary sliding at intermediate temperatures [1]. However, the formation of the terminal $\gamma / \mathrm{NbC}$ eutectic phase expands the solidification temperature range, which can result in increased susceptibility to weld solidification cracking [2]. Tantalum (Ta), as an alternative eutectic forming element, has been shown to increase the resistance to DDC and solidification cracking in the Ni-30Cr filler metals $[3,4]$. The present work aims to identify the eutectic constituents that form in the newly developed Ta-bearing filler metal 52XL using S/TEM. Previous work showed the formation of the MC carbide type phase using X-ray diffraction. However, scanning electron microscopy indicated the presence of another eutectic constituent highly enriched in chromium and molybdenum (Mo) [4]. Mo is added to $\mathrm{Ni}-30 \mathrm{Cr}$ alloys as a solid-solution strengthening element promoting high temperature strength.

The nominal composition of the filler metal $52 \mathrm{XL}$ is Ni-30Cr-8Fe-4Ta-4Mo. The welding wire was melted using a tungsten electrode arc-melting device into a button-shaped sample. The cross section was polished and etched with $10 \%$ chromic acid for $2-10 \mathrm{~s}$ at $5 \mathrm{~V}$. The region of interest was at the top of the solidified button, where most of the eutectic constituent was found. TEM samples from the eutectic interdendritic regions were prepared using Helios NanoLab ${ }^{\mathrm{TM}} 600$ DualBeam focused ion beam into thin foils. The specimens were further investigated using a Tecnai F20 S/TEM and an aberration corrected Titan 60-300 $\mathrm{S} / \mathrm{TEM}$. ED patterns were simulated using SingleCrystal ${ }^{\mathrm{TM}}$, CrystalMaker software.

Eutectic constituents were investigated using STEM and EDS mapping, as shown in Figure 1. The highangle annular dark field (HAADF) image shows the skeletal Ta-rich carbide (TaC) (bright region) and the $\gamma$ matrix (dark region). EDS mapping revealed a Cr- and Mo-rich phase in the left-bottom of Figure 1. Selected area diffraction (SAD) identified hexagonal (C14) Laves phase. Figure 2 shows the selected area diffraction pattern (SADP) under [1 $1 \overline{1} 0]$ zone axis and the simulated diffraction pattern using SingleCrystal ${ }^{\mathrm{TM}}$. The additional spots in the diffraction pattern are a result of double diffraction due to the large sample thickness. The specimens were further investigated by high-resolution imaging showing the atomic arrangement of the $\gamma$ matrix, Laves phases and MC carbide. The atomic positions of Laves phase were clarified. 


\section{References:}

[1] A Ramirez, JC Lippold, Mater. Sci. Eng. A, 380, no. 1-2, (2004) p. 245-258.

[2] J Dupont, C Robino, A Marder, Welding Journal, 77 (10), (1998) p. 417S-31S.

[3] AT Hope, JC Lippold, Welding in the World, 61 (2), (2017) p. 325-32.

[4] C Fink et al, ASME Pressure Vessels and Piping Conference, 6B, (2017) PVP2017-66130, 6 pages.

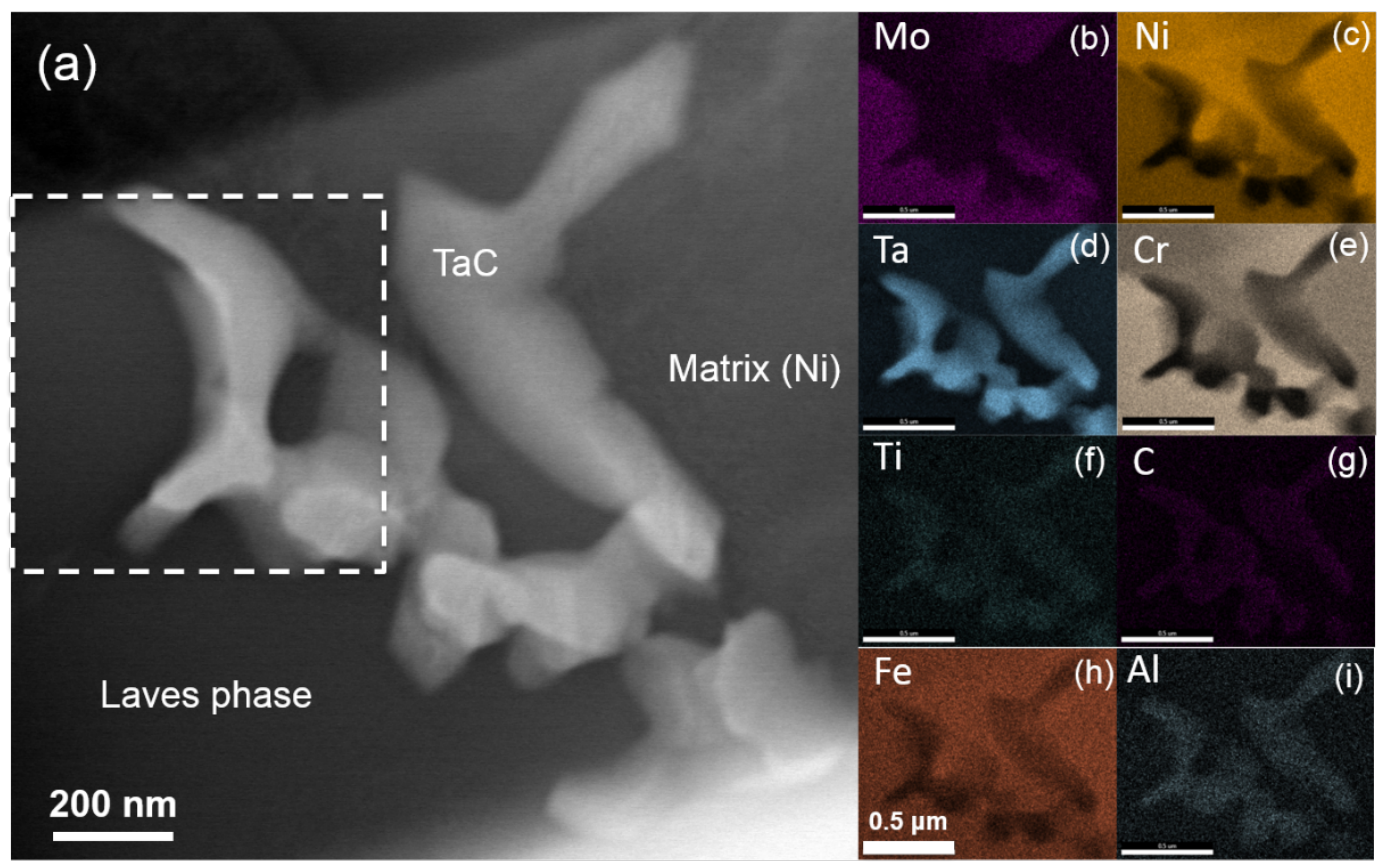

Figure 1. (a) The HAADF STEM image shows different contrast of eutectic constituents. (b)-(i) EDS mapping identifies the Ta-rich MC carbide depleted in Fe, Ni and $\mathrm{Cr}$, and $\mathrm{Mo}$ - and $\mathrm{Cr}$-rich Laves phase. The region for the selected area diffraction is indicated by the square in Figure 1 (a).
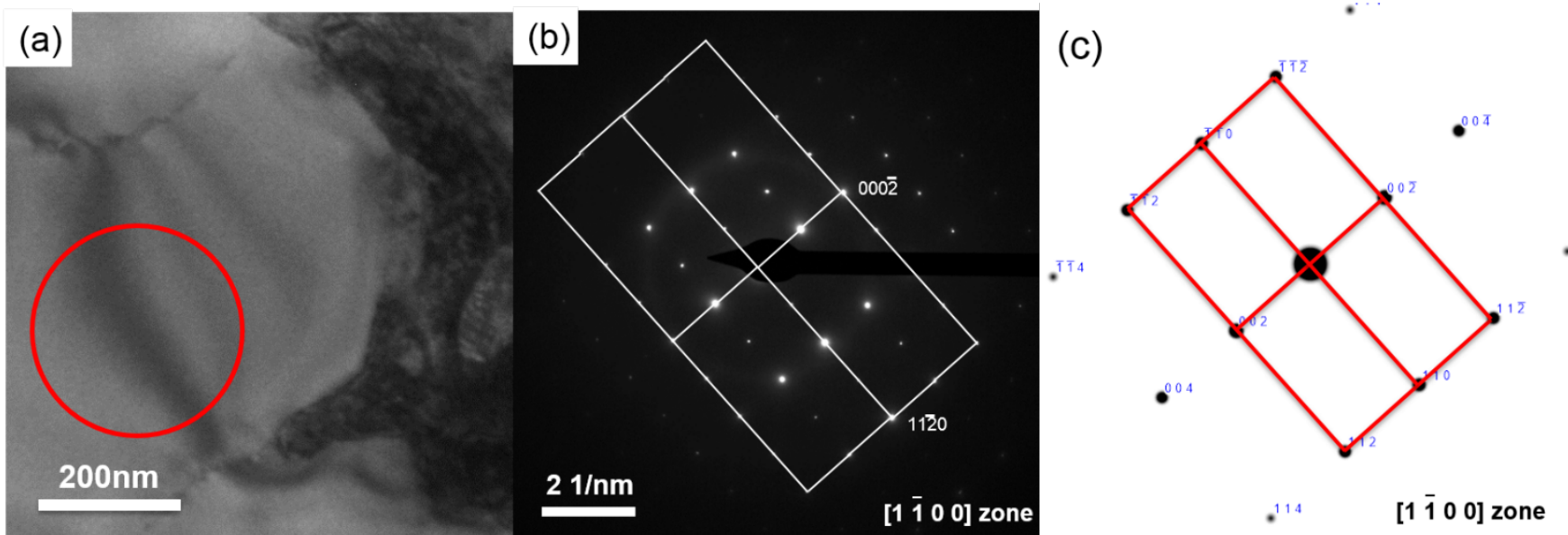

Figure 2. (a) Bright field image of eutectic constituent, (b) SADP of Laves phase (red circle) and (c) simulated diffraction pattern using SingleCrystal ${ }^{\mathrm{TM}}$. 